\title{
Teleradiology network system using the web medical image conference system with a new information security solution
}

Hitoshi Satoh, Noboru Niki, Kenji Eguchi, Hironobu Ohmatsu, Masahiro Kusumoto, et al.

Hitoshi Satoh, Noboru Niki, Kenji Eguchi, Hironobu Ohmatsu, Masahiro Kusumoto, Masahiro Kaneko, Ryutaru Kakinuma, Noriyuki Moriyama, "Teleradiology network system using the web medical image conference system with a new information security solution," Proc. SPIE 8319, Medical Imaging 2012: Advanced PACS-based Imaging Informatics and Therapeutic Applications, 83190Y (28 February 2012); doi: 10.1117/12.911431 


\title{
Teleradiology network system using the web medical image conference system with a new information security solution
}

\author{
Hitoshi Satoh 1), Noboru Niki 2), Kenji Eguchi 3), \\ Hironobu Ohmatsu4), Masahiko Kusumoto ${ }^{4)}$, Masahiro Kaneko5), Ryutaro Kakinuma ${ }^{5)}$, Noriyuki Moriyama ${ }^{5)}$, \\ Healthcare Informatics, Faculty of Healthcare, Tokyo Healthcare University, Japan 1) \\ Department of Optical Science, University of Tokushima, Japan 2) \\ School of Medicine, Teikyou University, Japan 3), National Cancer Center Hospital East, Japan 4) \\ National Cancer Center Hospital, Japan 5)
}

\begin{abstract}
We have developed the teleradiology network system with a new information security solution that provided with web medical image conference system. In the teleradiology network system, the security of information network is very important subjects. We are studying the secret sharing scheme and the tokenization as a method safely to store or to transmit the confidential medical information used with the teleradiology network system. The confidential medical information is exposed to the risk of the damage and intercept. Secret sharing scheme is a method of dividing the confidential medical information into two or more tallies. Individual medical information cannot be decoded by using one tally at all. Our method has the function of automatic backup. With automatic backup technology, if there is a failure in a single tally, there is redundant data already copied to other tally. Confidential information is preserved at an individual Data Center connected through internet because individual medical information cannot be decoded by using one tally at all. Therefore, even if one of the Data Centers is struck and information is damaged due to the large area disaster like the great earthquake of Japan, the confidential medical information can be decoded by using the tallies preserved at the data center to which it escapes damage. Moreover, by using tokenization, the history information of dividing the confidential medical information into two or more tallies is prevented from lying scattered by replacing the history information with another character string (Make it to powerlessness). As a result, information is available only to those who have rightful access it and the sender of a message and the message itself are verified at the receiving point. We propose a new information transmission method and a new information storage method with a new information security solution.
\end{abstract}

Keyword : MDCT, computer-aided diagnosis, web medical image conference, cryptography, secret sharing scheme, teleradiology, tokenization, token, data center, cloud computing, internet

\section{INTRODUCTION}

The invention of computers made the need for security of digital information a critical issue. Advances in the field of computer networks have made information security even more important Computer systems have to be equipped with mechanisms for security data. Computer networks need provisions that secure data from possible intrusions. Network security can be divided into two broad categories: cryptographic techniques and authentication techniques (verification). Network security is top-priority in data networks. As communication networks are growing rapidly, security issues have pushed to the forefront of concern for end users, administrators, and equipment suppliers.

Network security is concerned mainly with the following two elements:

1. Confidentiality. Information should be available only to those who have rightful access to it.

2. Authenticity and integrity. The sender of a message and the message itself should be verified at the receiving point.

*Correspondence: Email:h-sato@thcu.ac.jp; Telephone:+81-3-5799-3712

Medical Imaging 2012: Advanced PACS-based Imaging Informatics and Therapeutic Applications,

edited by William W. Boonn, Brent J. Liu, Proc. of SPIE Vol. 8319, 83190Y · @ 2012 SPIE

CCC code: $1605-7422 / 12 / \$ 18 \cdot$ doi: $10.1117 / 12.911431$

Proc. of SPIE Vol. $831983190 Y-1$ 
Common solutions that can protect computer communication networks from attacks are classified as cryptographic techniques or authentication techniques. Today, network engineers focus on cryptography methods for computer communication networks. Cryptography is the process of transforming a piece of confidential medical information or message shared by two parties into some sort of code. One of the key technologies to enable a PACS is computer communication networks. Computer communication networks need the process of transforming the confidential medical information or message into some sort of code for cryptography. Primitive Local Area Networks were used for Picture Archiving and Communications System (PACS), and point-to-point links were used for teleradiology systems.

A lot of years ago, when the engineering world was transmitting billions of bits of data around the world, teleradiology was viewed as transmitting medical image away from the modality. Teleradiology needs the process of transforming the confidential medical information or message into some sort of code for cryptography, too. Cryptography is the process of transforming a piece of information or message shared by two parties into some sort of code. Encrypted information can surely be decoded by all the expressions that can be obtained by using the super computer.

Because of such a background, we are studying the secret sharing scheme and tokenization as a new information security solution to store or to transmit the confidential medical information. In addition, we studies PACS and teleradiology systems with a new information security solution for a computer-aided diagnosis (CAD) system.

\section{ALGORITHM}

Diagnostic MDCT imaging requires a considerable number of images to be read. It is this time-consuming step that makes the use of helical MDCT impractical at present. This section describes the secret sharing scheme as a new information security solution to store or to transmit the confidential medical information, and the four main features of our computer-aided chest diagnosis system: an algorithm that detects suspected lung cancers in CT images; an algorithm that detects suspected coronary artery calcification; a multiplanar reconstruction technique that displays arbitrary cross sections of CT images; and a graphical three-dimensional method that facilitates image registration and fusion, and provides quantitative geometric and volume.

\subsection{Diagnostic Algorithm}

\subsubsection{Automated Detection of Lung Cancer Nodules}

The algorithm for the detection of candidate lung cancer regions was applied to the helical CT images of 500 subjects (total: 15750 images). The sensitivity of automatic detection of nodules was about 95\%. Figure 1 shows the helical CT image. Figure 2 shows an example of the automatic detection results of the CAD system. Figure 3 shows the algorithm of CAD system The algorithm for the detection of candidate lung cancer regions was applied to the helical CT images of 500 subjects (total: 15750 images). The sensitivity of automatic detection of nodules was about $94 \%$. The suspected lung cancer detection algorithm was applied to 500 subjects. The 309 shadows in 500 subjects contained suspected lung cancers. When a screening physician who cooperated in the study read about CT images without the assistance of a computer-aided diagnosis (CAD) system, 245 of these 309 shadows were true positive (TP) and 64 were false negative $(\mathrm{FN})$. When the same screening physician performed image diagnosis with the assistance of a CAD system in which the suspected lung cancer detection algorithm was installed, 290 of the shadows were TP and 19 were FN. In other words, 45 suspected lung cancers that were missed by a physician without the assistance of the CAD system were identified as positive lesions in diagnosis by the same physician with the assistance of the CAD system. This finding suggests that diagnostic accuracy can be increased if physicians perform diagnosis with the assistance of a CAD system. We applied this algorithm to 50 subjects(total:150 CT scans). The combination number of the comparison of each slice image in the present and the early CT scans was 100 (total:2,806 slice images). This algorithm could compare correctly the slices in 90 (total:2,734 slice images) of 100 CT scans combination with respect to physician's point of view. Physician's point of views are the region of the blood vessel and the bronchia tube with respect to each slice image. 


\subsubsection{Automated Detection of Coronary Artery Calcification}

The $\mathrm{CT}$ images acquired in mass screening for lung cancer, which include the entire lung field, were also analyzed using the suspected coronary artery calcification detection algorithm. Figure 4 shows the coronary artery calcification detection algorithm. The uppermost slice position was determined based on the distance between the right and left lobes of the lungs, the positions of the clavicles, and the shape and area of the heart. The lowermost slice position was determined based on the position of the diaphragm. Since the courses of the coronary arteries in each slice were known, the slices were grouped into three zones including the various courses of the coronary arteries based on the shape of the heart, density distribution, position of the trachea, and bony structures.

We exclude the artifact regions by using the diagnostic rules. The diagnostic rules are constructed from two rules-Rule 1 and Rule2. First, if the candidate area meets the condition of Rule1, we regard it as an artifact. Second, we apply the candidate regions which are not judged as artifacts in Rule1, to a neural network. We separate the candidate regions intothe artifacts and calcifications.

The results obtained when the suspected coronary artery calcification detection algorithm was applied to about 16,000 CT images obtained from 462 subjects. An expert physician diagnosed all the data of coronary artery calcification by the criterion. Shadows classified as category E are strongly suspected to indicate calcification of the coronary artery and therefore require detailed examination. Shadows classified as category D are those for which coronary artery calcification cannot be ruled out. 214 shadows in the 16,000 CT images were suspected to indicate coronary artery calcification and that the CAD system with the suspected coronary artery calcification detection algorithm correctly identified all of the 91 shadows in category E (100\% detection) as well as 122 of the 123 shadows in category D (99.2\% detection).

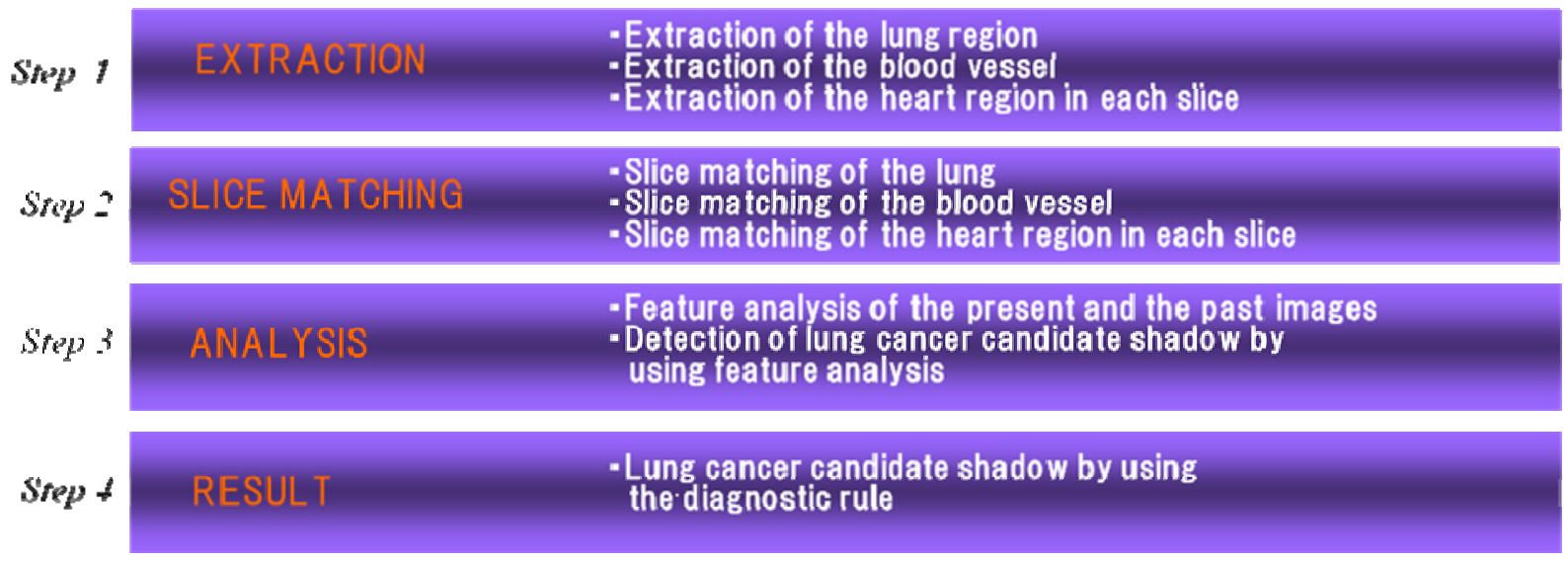

Figure 3 Flowchart of Automated Detection of Lung Cancer Nodules.

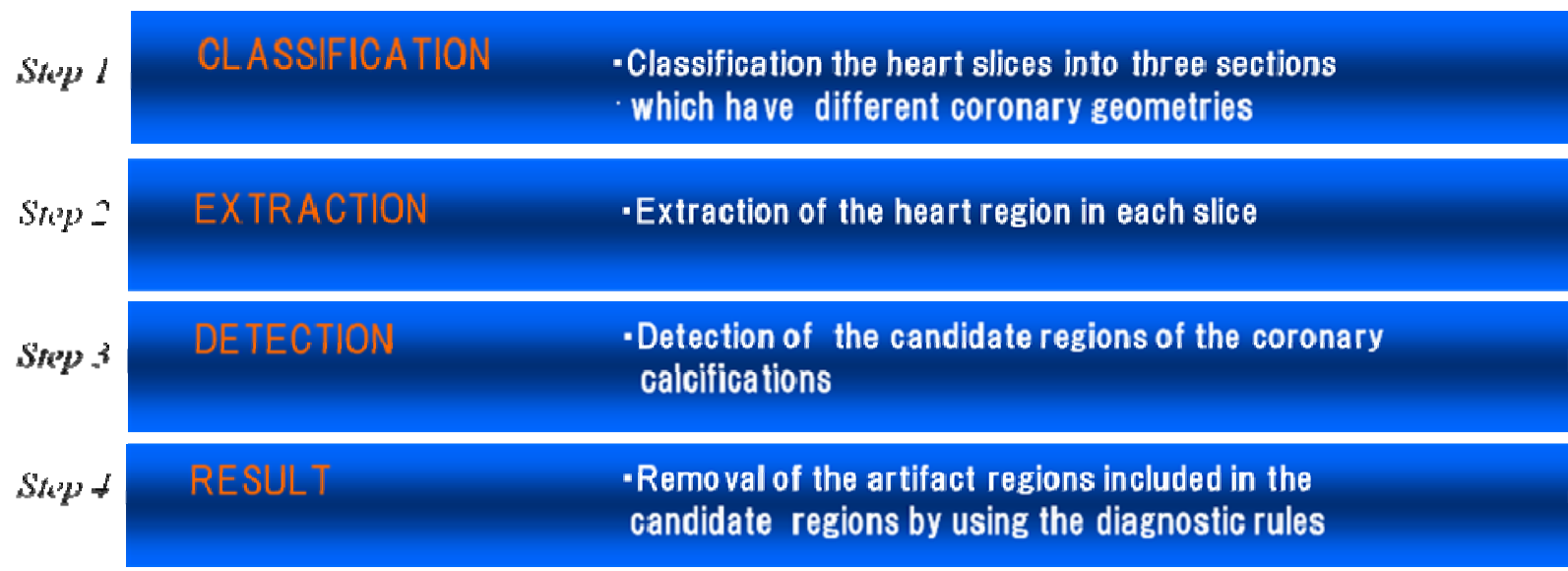

Figure 4 Flowchart of diagnostic algorithm of coronary calcification. 


\subsection{Security Algorithm}

\subsubsection{Secret Sharing Scheme}

Secret sharing scheme is a method of dividing the confidential medical information into two or more share. There are perfect secret sharing scheme and threshold secret sharing scheme in the kind of the technique of secret sharing scheme. Our method is called perfect secret sharing scheme. Perfect secret sharing scheme can encode the confidential information to two or more share. However, when all share cannot be collected, the confidential information cannot be decoded. Figure 5 shows the procedure when perfect secret sharing scheme encodes manuscript to three shares. Figure 6 shows the procedure when perfect secret sharing scheme decodes three encoded shares to the manuscript. Figure 7 shows the procedure when threshold secret sharing decode to the manuscript by using two of three encoded shares. This figure shows that the manuscript can be decoded by using only two set of share even if one of three share loses. Figure 8 shows the procedure of perfect secret sharing scheme.

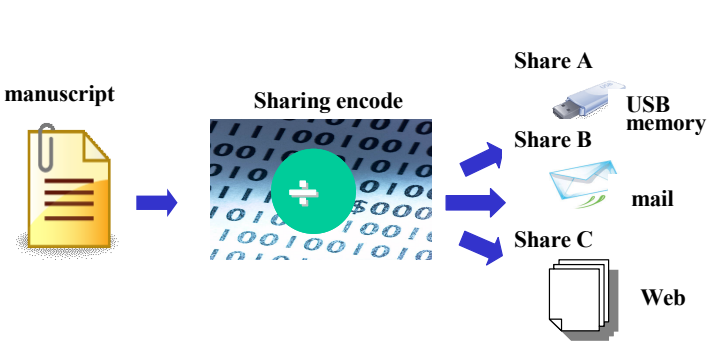

Figure 5. Secret sharing procedure.

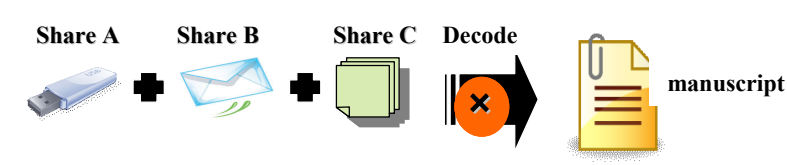

Figure 6. Perfect secret sharing procedure.

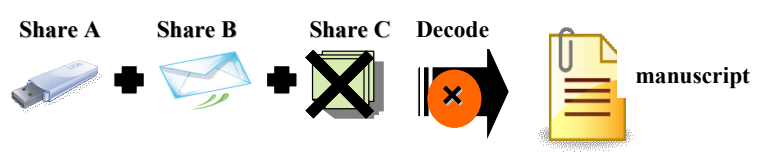

Figure 7. Threshold secret sharing decode procedure.

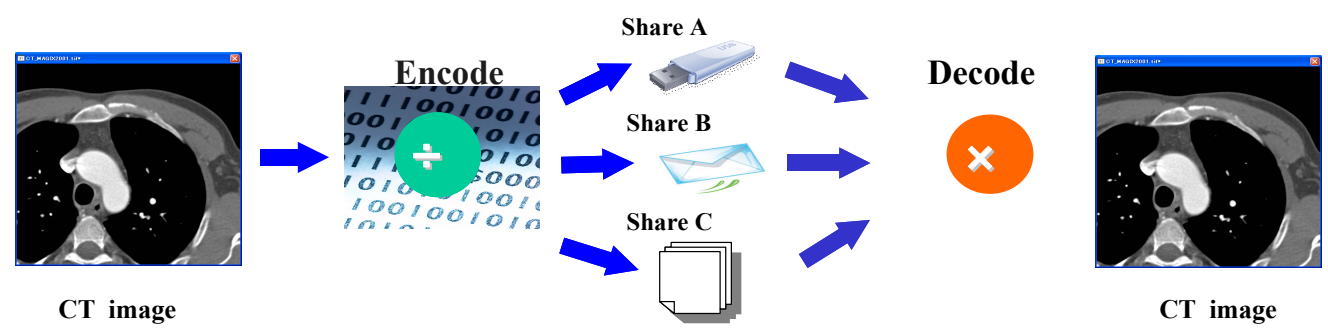

Figure 8. Configuration of perfect secret sharing scheme.

\subsubsection{Secret Sharing Procedure}

Common solutions that can protect computer communication networks from attacks are classified as cryptographic techniques or authentication techniques. Today, network engineers focus on cryptography methods for computer communication networks. Cryptography is the process of transforming a piece of confidential information or message shared by two parties into some sort of code. Secret sharing scheme is a method of dividing this confidential information into two or more share. Figure 9 and Figure 10 show the algorithm in the software of the perfect secret sharing scheme of Toppan Printing Co., Ltd that we are using. This software provides the function to divide original information into digital non-linear data of each bit. The data divided nonlinearly forms two shares of $\mathrm{F}(\mathrm{X})$ and $\mathrm{F}(\mathrm{Y})$. To prevent tapping and the falsification, these $\mathrm{F}(\mathrm{X})$ and $\mathrm{F}(\mathrm{Y})$ are kept in separate storage. This software is called "GFI E-Tally". Our network system can perform Web medical image conference in the medical institutions of a remote place using the web medical image conference system with information security solution called "GFI E-Tally. 


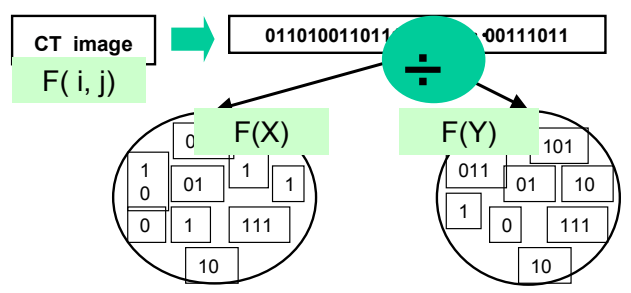

Figure 9. Algorithm of perfect secret sharing scheme.

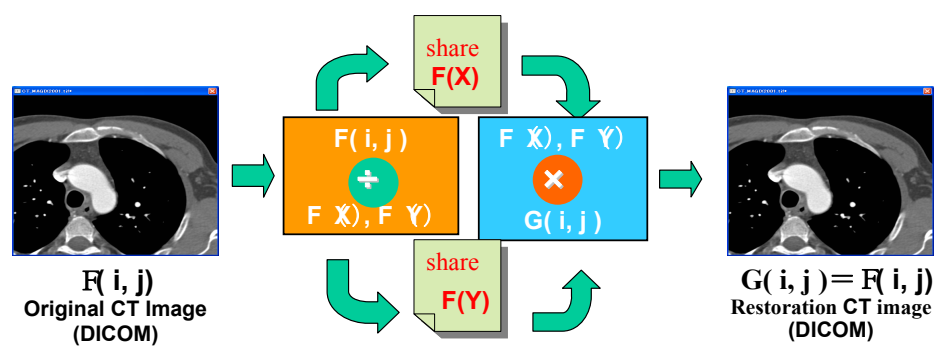

Figure 10. Configuration of perfect secret sharing.

\subsubsection{Network security}

Today, network engineers focus on cryptography methods for computer communication networks. Cryptography is the process of transforming a piece of confidential medical information or message shared by two parties into some sort of code. We are studying the secret sharing scheme as a method safely to store or to transmit this confidential medical information. Secret sharing scheme is a method of dividing the confidential medical information into two or more share. Our secret sharing method is different from the cryptography methods because original data cannot be decoded from one file divided by a secret sharing method at all. Therefore, knowledge concerning the medical image doesn't leak at all even if share file is stolen. Our method has both perfect and threshold secret sharing communication functions. Perfect secret sharing scheme can encode the confidential medical information to two or more share. Our network system can perform web medical image conference in the medical institutions of a remote place. Figure 11 shows two share file $F(X)$ and $\mathrm{F}(\mathrm{Y})$ sent by using a separate forwarding route from client A for client B. Figure 12 shows the case of three share file $\mathrm{F}(\mathrm{X}), \mathrm{F}(\mathrm{Y})$ and $\mathrm{F}(\mathrm{Z}) . \mathrm{F}(\mathrm{X}), \mathrm{F}(\mathrm{Y})$ and $\mathrm{F}(\mathrm{Z})$ are share files encoded by using the software called "GFI E-Tally". If all these share files are obtained, the medical information is completely restorable. However, knowledge is not obtained for the medical information quite at all if it lacks even by one of these share files. Moreover, the software called "GFI E-Tally" has the function of threshold secret sharing scheme. With RAID technology, we can be decoded by using only remaining share if one of some shares loses by the damage.

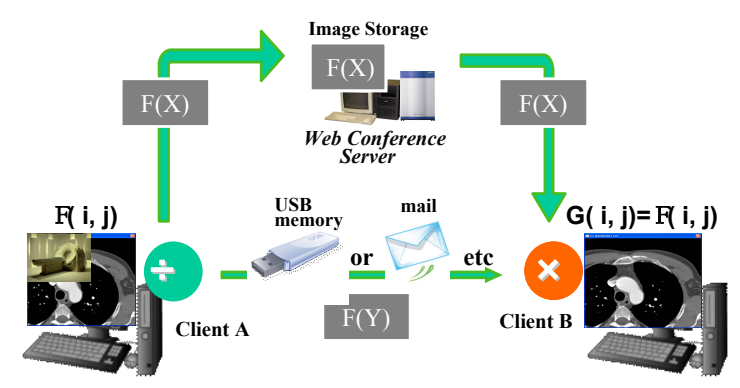

Figure 11. Secret sharing communication system with two channels: $\mathrm{F}(\mathrm{X})$ and $\mathrm{F}(\mathrm{Y})$.

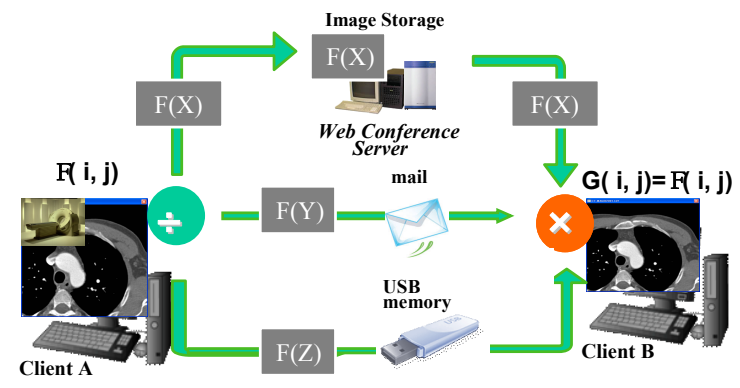

Figure 12. Secret sharing communication system with three channels: $F(X), F(Y)$ and $F(Z)$. 


\subsubsection{Network security and RAID Technology}

Figure 13. shows the case of three share file $\mathrm{F}(\mathrm{X}), \mathrm{F}(\mathrm{Y})$ and $\mathrm{F}(\mathrm{Z})$ that encode the DICOM medical image data by using the software called "GFI E-Tally". These files are exposed to the risk of the damage and intercept. Figure 14. shows the concept of image transfer to remote sites using PACS. The use of Redundant Array of Independent Disks (RAID) technology and back-up strategies are some of the techniques used to avoid loss data. Replacement parts for archive components must be kept on-site or close enough for very rapid swap out when equipment fails, and of course someone must be available who can repair or replace damaged equipment. We can not be decoded by using only two set of share if one of three share loses by the damage in the case of Figure 13. Three data centers as shown in Figure 14 has the function of RAID because this problem is solved. With RAID technology, if there is a failure in a single database of three data centers, there is redundant data already copied to other data center. Table 1. shows RAID type and number of encoded secret sharing file by using our web medical image conference system with a new information security solution. In addition, it is not possible to decode by using one encoded secret sharing file even if the file is intercepted in our system.

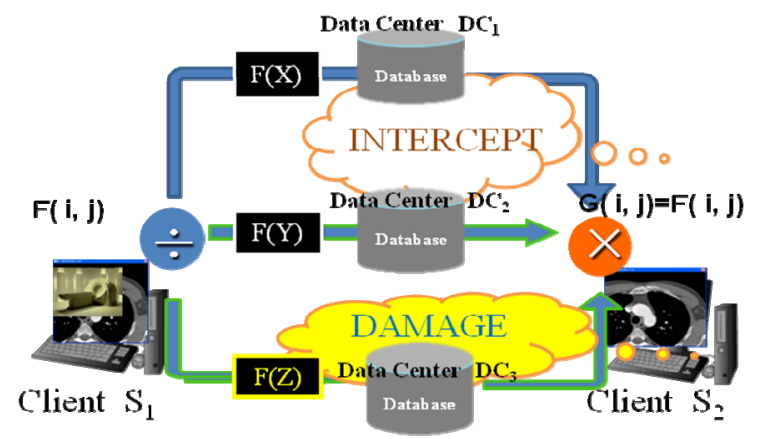

Figure 13. Threshold secret sharing communication system with three channels: $F(X), F(Y)$ and $F(Z)$
TABLE 1. RAID type and secret sharing scheme

\begin{tabular}{|c|c|c|c|}
\hline $\begin{array}{c}\text { RAID type } \\
\text { Number } \\
\text { of shares }\end{array}$ & $N$ & N-1 & $N \cdot \mathbf{z}$ \\
\hline 3 & 0 & 0 & - \\
\hline 5 & 0 & 0 & - \\
\hline 7 & 0 & 0 & 0 \\
\hline 10 & 0 & 0 & 0 \\
\hline
\end{tabular}

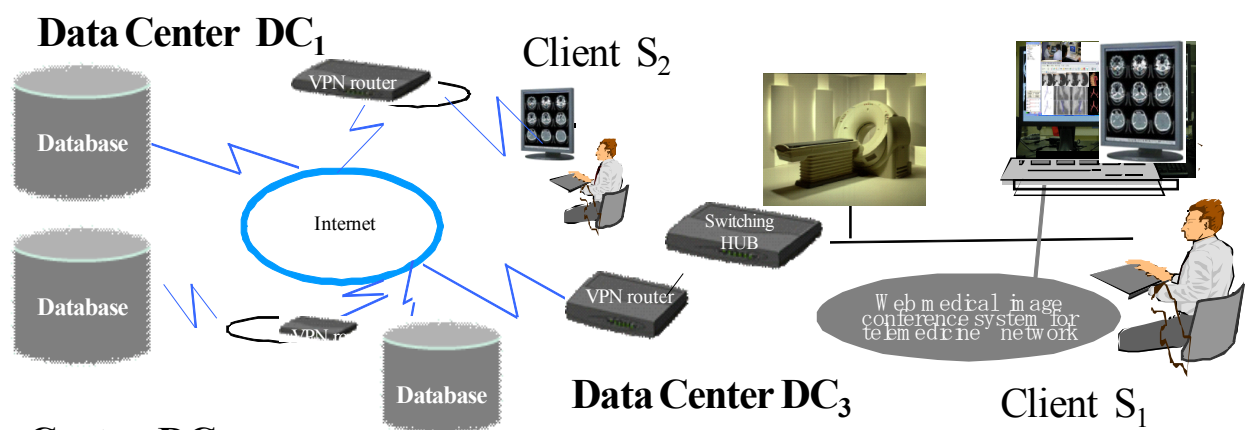

Data Center $\mathrm{DC}_{2}$

Figure 14. RAID type and Data Center of internet 


\subsection{Data Tokenization and History Information}

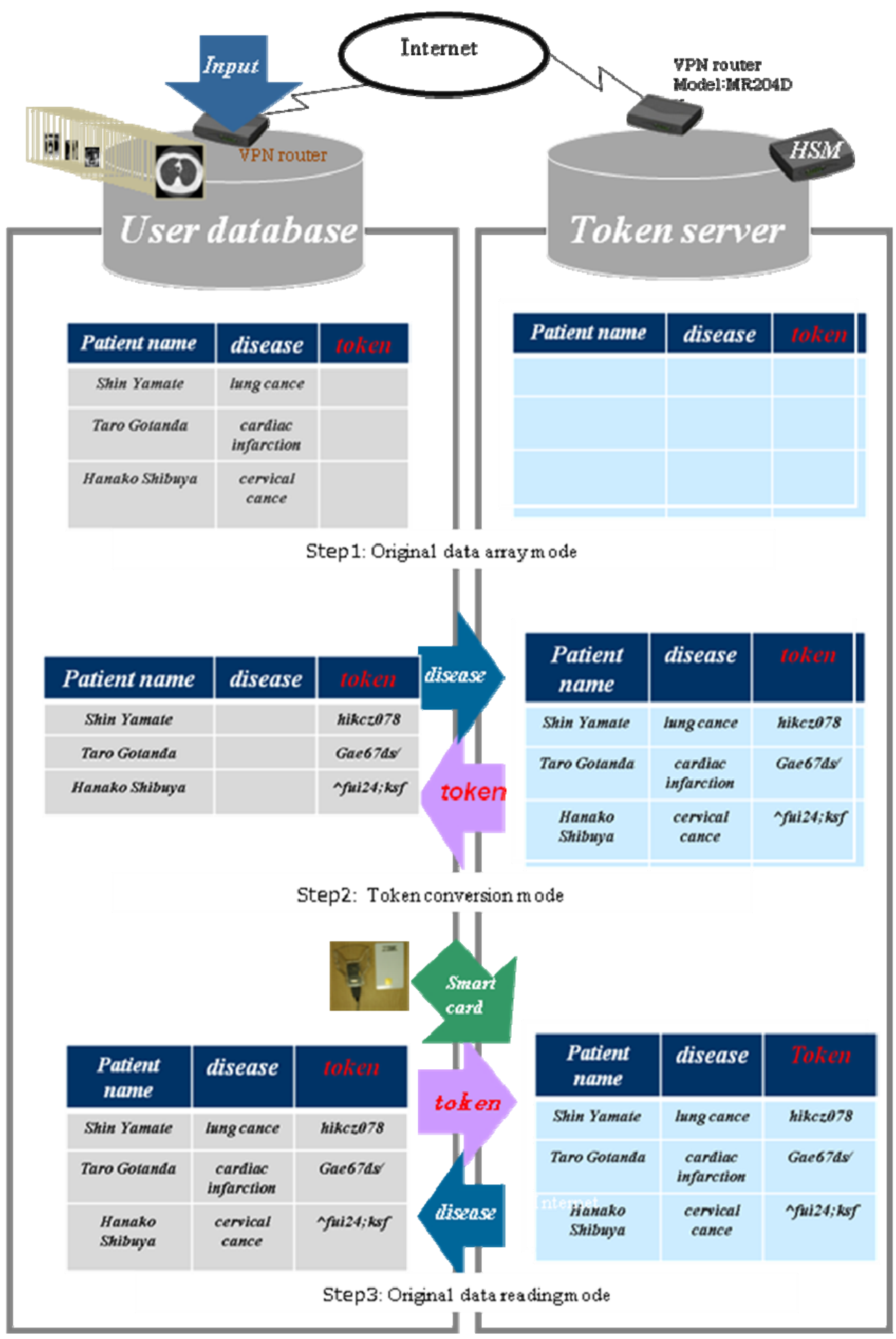

Figure 15. User database and Token server. 


\subsubsection{Data Tokenization}

Data Tokenization doesn't change the data form, and be a technology in the data of the fake that nullifies replace important as for the critical information on individual information etc. Figure. 15 shows algorithm of tokenization. Tokenization system consists of User database and Token server. Step 1, 2, and 3 show the procedure of tokenization. Step 1 is original data array of user database. Step 2 shows the state that original data is converted into Token. Step 3 shows the state to read original data from token server by using Token and smart card in accordance with PKI.

\subsubsection{History Information}

When our treleradiology network system with secret sharing scheme is used, share files are distributed to two or more data centers mutually and geographically away and is preserved. Moreover, it is necessary to collect all share files preserved at each data center to one place again to decode original information. It is necessary to record an address at each data center on the Internet, modality of image, and information on the number of share file etc. beforehand to decode later. We call this information history information of secret sharing scheme. In addition, the history information on teleradiology database is changed for another meaningless character string by applying tokenization.

Figure. 16 shows the example of replacing important history information on the teleradiology database with a meaningless Token with the Token server. Here, Token server uses a secret code key basic technology that builds in HSM that can defend even national secret information from the illegal computer access. We can read the original data of history information of secret sharing scheme from the Token server by using Token and smart card in accordance with PKI.

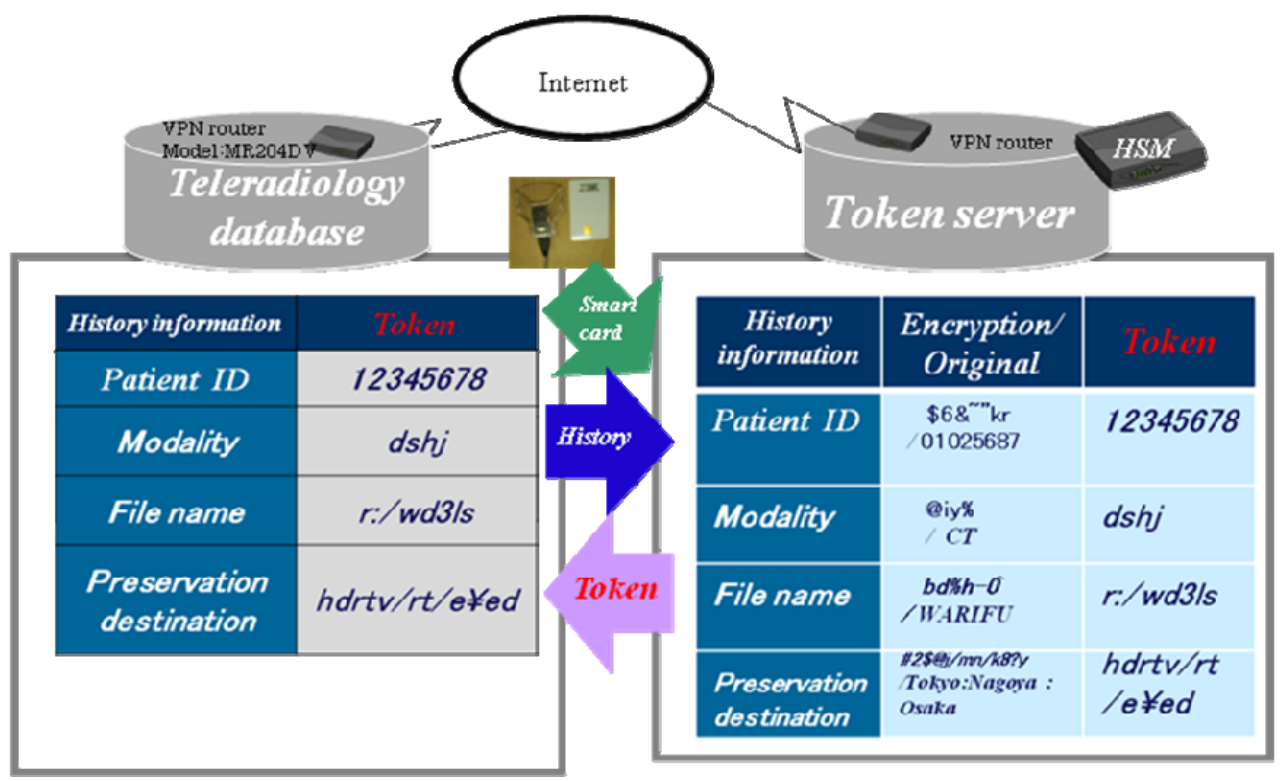

Figure 16. History information of secret sharing scheme 


\section{SYSTEM}

Teleradiology is one of the most well-developed applications of telemedicine. Common solutions that can protect computer communication networks from attacks are classified as cryptographic techniques or authentication techniques. Today, network engineers focus on cryptography methods for computer communication networks. Our network experiment system can perform Web medical image conference in the medical institutions of a remote place using the web medical image conference system with a new information security solution called secret sharing scheme as shown in Figure 17. Secret sharing scheme is a method of dividing the confidential medical information into two or more tallies. We can safely share the screen of computer-aided diagnosis workstation to which the medical image of Data Center is displayed from two or more web conference terminals at the same time. The history information of secret sharing scheme on teleradiology database is changed for another meaningless character string by applying tokenization. As a result, information is available only to those who have rightful access it and the sender of a message and the message itself are verified at the receiving point

In addition, the use of Redundant Array of Independent Disks (RAID) technology and back-up strategies are some of the techniques used to avoid loss data. Real time biometric face authentication system is connected with CAD image filing system shown in Figure 17. Real time biometric face authentication system analyzes the feature of the face image of which it takes a picture in 20 seconds with the camera and defends the safety of the medical information. Real time biometric face authentication system makes "Success in login" and "Encryption of file" used on site of teleradiology effective.

\subsection{Teleradiology network configuration}

The teleradiology network system uses Web medical image conference system. With RAID technology, if there is a failure in a single database of three data centers as shown in Figure 17, there is redundant data already copied to other data center. The screen is shared from two or more web conference terminals at the same time. An opinion each other can be exchanged mutually by using the camera and the microphone connected with web conference terminal.

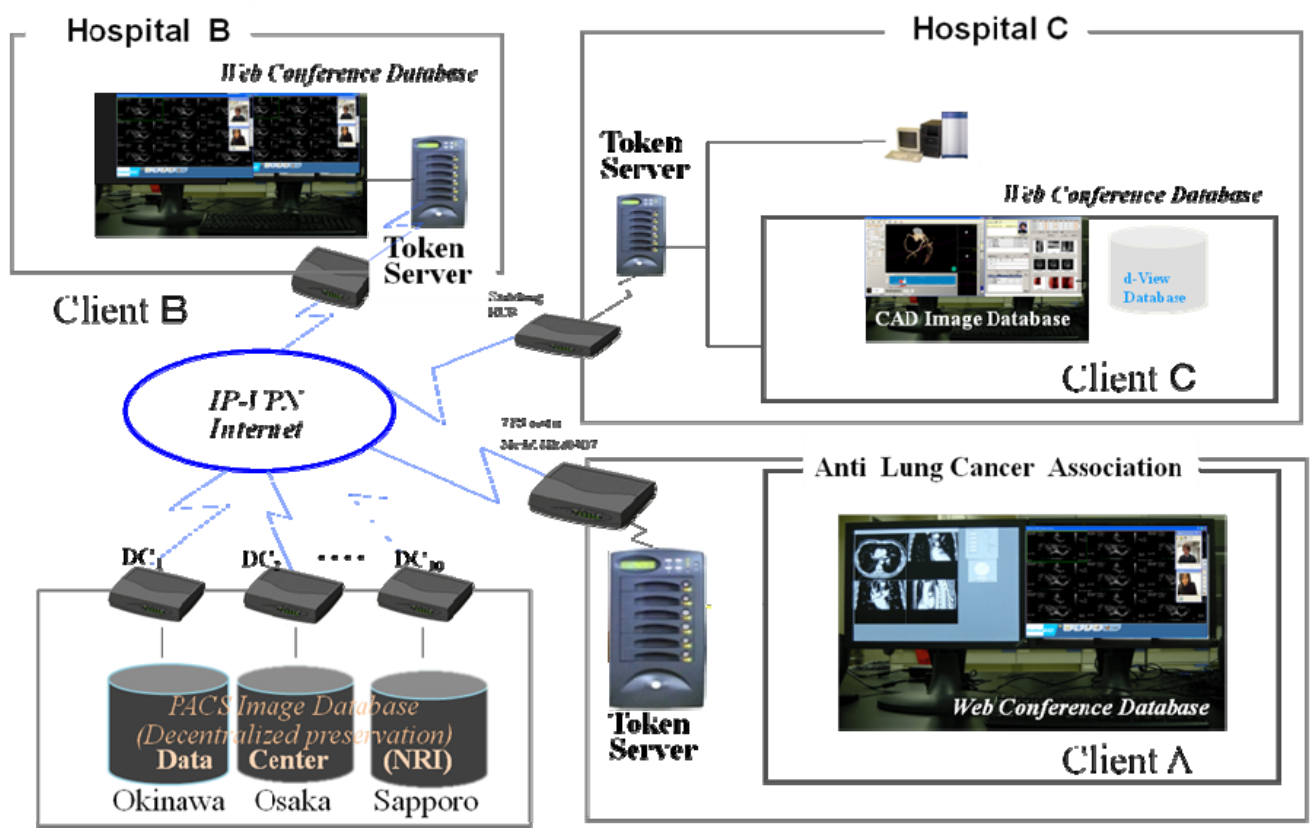

Figure 17. Teleradiology network experiment system by using Web medical image conference system:ViewSend RAD. 


\subsection{Security of confidential medical information}

\subsubsection{Personal authentication}

Common solutions that can protect computer communication networks from attacks are classified as cryptographic techniques or authentication techniques. Real time biometric face authentication system as shown in Figure 18. is connected with Data Center of GSD. Real time biometric face authentication system analyzes the feature of the face image of which it takes a picture in 20 seconds with the camera and defends the safety of the medical information. Real time biometric fingerprint authentication system and real time biometric face authentication system makes "Success in login" and "Encryption of file" effective by the interaction. We use the web browser operation of the real time biometric face authentication system and start the web medical image conference terminals.

\subsubsection{Network Security and Encryption}

Today, network engineers focus on cryptography methods for computer communication networks. However, Secret sharing scheme that we are proposing is a method different from the encryption technology. Secret sharing scheme is a method of dividing the confidential medical information into two or more shares. Confidential Medical information passes a different information transportation route. Our network experiment system can perform Web medical image conference to improve safety by secret sharing scheme called "GFI E-Tally". Figure 19. shows the web medical image conference terminals (1),(2) and five Data Center of NRI connected through a tunnel using IP-VPN internet. Figure 20. shows indicator of decentralized preservation. In addition, the use of Redundant Array of Independent Disks (RAID) technology and back-up strategies are some of the techniques used to avoid loss data. Replacement parts for archive components must be kept on-site or close enough for very rapid swap out when equipment fails, and of course someone must be available who can repair or replace damaged equipment. We can not be decoded by using only four set of share if one of five shares loses by the damage. Five data centers have the function of RAID because this problem is solved. With RAID technology, if there is a failure in a single database of five data centers, there is redundant data already copied to other data center. We can share the screen of workstation to which a medical image of a center medical institution is displayed from two or more web conference terminals at the same time. Moreover, an opinion each other can be exchanged mutually by using a camera and a microphone connected with workstation. These voice and all data like medical images are encrypted. As a result, the safety of the medical information is secured.

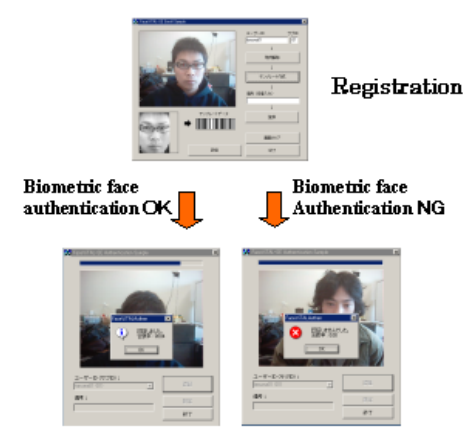

Figure 18. Biometric face authentication system.

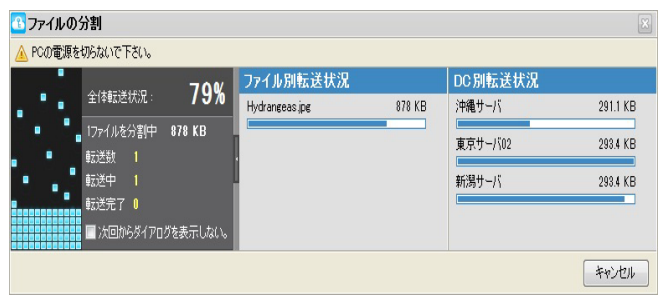

Figure 20. Indicator of Decentralized preservation

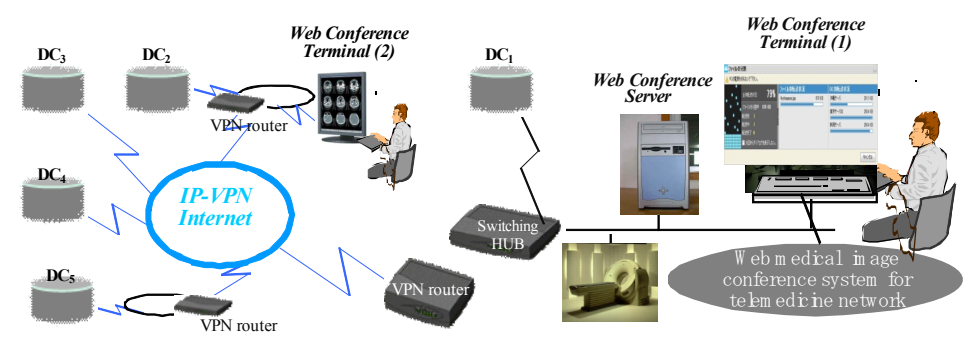

Figure 19. Web medical image conference system with a new information security solution. 


\section{RESULTS}

We are studying the secret sharing scheme and the tokenization as a method safely to store or to transmit the confidential medical information used with the teleradiology network system. The confidential medical information is exposed to the risk of the damage and intercept. Secret sharing scheme is a method of dividing the confidential medical information into two or more tallies. We can safely share the screen of computer-aided diagnosis workstation to which the medical image of Data Center is displayed from two or more web conference terminals at the same time. There are following that

1) the web medical image conference system by using information security solution called secret sharing scheme and tokenization

2) each divided confidential information(tally) preserved at an individual Data Center connected through internet

3) Data Centers (cloud computing) that provides with RAID function

4) because the backup function is provided even if the large area disaster occurs, the worry of the loss of the medical information data is unnecessary

5) the history information of secret sharing scheme on teleradiology database is changed for another meaningless character string by applying tokenization

6) as a result, information is available only to those who have rightful access it and the sender of a message and the message itself are verified at the receiving point

\section{CONCLUSIONS}

The results of this study indicate that our teleradiology network system can increase diagnostic speed, diagnostic accuracy, and personal information protection by using secret sharing scheme, tokenization, personal authentication, IP-VPN internet and Encryption. As teleradiology network system gain more widespread acceptance in the near future, it is expected that physicians involved in community health will be able to read a considerable number of images with the assistance of computer-aided diagnosis (CAD) workstation and teleradiology network system under a safe infrastructure.

\section{ACKNOWLEDGEMENTS}

We would like to thank GFI, NRI, Toppan, Japan SafeNet and View Send ICT who are our research cooperator for providing the sofutoware of secret sharing scheme, Token server, and smart card. We would like to thank View Send ICT Corporation who is our research cooperator for providing the Web medical image conference system.

\section{REFERENCES}

1. N.Niki, Y.Kawata, H.Satoh: A 3-D Display Method of Fuzzy Shapes Obtained from Medical Images, Trans.IEICE, Japan, vol.J73-D-II,no.10,pp.1707-1715, 1990.

2. K.Kanazawa, N.Niki, H.Nishitani, H.Satoh, H.Omatsu, N.Moriyama, "Computer Assisted Diagnosis of Lung Cancer Using Helical X-ray CT,” IEEE Computer Society Press, pp.261-267,1994.

3. M.Kaneko, K.Eguchi, H.Hirano, H. Ohmatsu, and the Anti-Lung Cancer Association(ALCA) Tokyo, Chest helical CT (CHCT), " a utility in the early detection of peripheral lung cancer (PLC), “ Am. J. Resp. Critical Care Med., vol.154, p.A772, 1995.

4. K.Kanazawa, K.Kubo, N.Niki, H.Satoh, H.Ohmatsu, K.Eguchi, N.Moriyama, "Computer Aided Screening System for Lung Cancer Based on Helical CT Images," Visualization in Biomedical Computing, Lecture Notes in Computer Science, Springer, pp.223-228, 1996.

5. K.Kanazawa, H.Satoh, M.Kubo, N.Niki, H.Ohmatsu, K.Eguchi, N.Moriyama, "Computer Aided Diagnosis for Lung Cancer Based on Helical CT Images of Mass Screening," 13th International Conf. on Pattern Recognition, Application and Robotic System, IEEE Computer Society Press, pp.381-385, 1996.

6. Y.Kawata,K.Kanazawa,S.Toshioka,N.Niki,H.Satoh,H.Ohmatsu,K.Eguchi,N.Moriyama, "Computer Aided Dignosis System for Lung Cancer Based on Helical CT Images", Lecture Notes in Computer Science 1311, Image Analysis and Processing, Springer, pp420-427,1997.

7. S.Toshioka, K.Kanazawa, N.Niki, H.Satoh, H.Ohmatsu, K.Eguchi, N.Moriyama, "Computer Aided Diagnosis 
System for Lung Cancer Based on Helical CT Images," Proc. SPIE Volume 3034 Part 2, pp.975-984, California, 1997.

8. K.Kanazawa, H.Satoh, Y.Kawata, N.Niki, H.Ohmatsu, R.Kakinuma, M.Kaneko, K.Eguchi, N.Moriyama, "Computer-Aided Diagnosis for Lung Cancer Based on Helical CT Images," proceedings of the Second IEEE International Conference on Intelligent Processing Systems, pp.27-31, 1998.

9. H.Satoh, Y.Ukai, N.Niki, H.Ohmatsu, R.Kakinuma, K.Mori, K.Eguchi, M.Kaneko, N.Moriyama, "Computer-Aided Diagnosis System for Lung Cancer Based on Retrospective Helical CT Images," SPIE Medical Imaging, San Diego,3661, pp.1324-1335, 1999.

10. H. Taguchi, H. Satoh, Y. Kawata, N. Niki, K. Eguchi, H. Ohmatsu, R. Kakinuma, M. Kaneko, N. Moriyama, "Lung cancer detection based on helical CT images using curved-surface morphology," SPIE Medical Imaging, San Diego, 3661-138, pp.1307-1314, 1999.

11. Y.Ukai, N.Niki, H.Satoh, S. Watanabe, K.Eguchi, H. Ohmatsu, N. Moriyama, "CAD system for coronary calcifications based on helical CT images," SPIE Medical Imaging, San Diego, 3661-139, pp.1315-1323, 1999.

12. Y.Ukai, N.Niki, H.Satoh, K.Eguchi, K.Mori, H.Ohmatsu, R.Kakinuma, M.Kaneko, N.Moriyama, "Computer aided diagnosis system for lung cancer based on retrospective helical CT images," SPIE Medical Imaging, San Diego, 3979, pp.1028-1039, 2000.

13. H.Satoh, Y.Ukai, N.Niki, K.Eguchi, K.Mori, H. Ohmatsu, R. Kakinuma, M. Kaneko, N. Moriyama, "Computer-Aided Diagnosis System for Comparative Reading of Helical CT Images for the Detection of Lung Cancer," IEICE Trans. on Information and Systems, ,VOL.E84-D, NO.1,pp.161-170, January, 2001.

14. H.Satoh, N.Niki, K.Mori, K.Eguchi, M.Kaneko, N. Moriyama, H.Ohmatsu, R.Kakinuma, H.Masuda, S.Machida,M.Sasagawa, "Innovation of PACS for computer aided diagnosis with functions to assist comparative reading for lung cancer based on multi-helical CT images," SPIE Medical Imaging, San Diego, VOL.5033, pp.413-423, 2003.

15. H.Satoh, N.Niki, K.Mori, K.Eguchi, M.Kaneko, N. Moriyama, H.Ohmatsu, R.Kakinuma, H.Masuda, S.Machida, M.Sasagawa, "Computer-aided diagnosis workstation and database system for chest diagnosis based on multihelical CT images," SPIE Medical Imaging, San Diego, VOL.5371, pp.402-412, 2004.

16. H.Satoh, N.Niki, K.Mori, K.Eguchi, M.Kaneko, N. Moriyama, H.Ohmatsu, R.Kakinuma, H.Masuda, S.Machida, M.Sasagawa, "Computer-aided diagnosis workstation for chest diagnosis based on multihelical CT images," SPIE Medical Imaging, San Diego, VOL.5748, pp.480-490, 2005.

17. H.Satoh, N.Niki, K.Mori, K.Eguchi, M.Kaneko, N. Moriyama, H.Ohmatsu, R.Kakinuma, H.Masuda, S.Machida, M.Sasagawa," Computer-aided diagnosis workstation and database system for chest diagnosis based on multihelical CT images,” SPIE Medical Imaging, San Diego,„Vol.6145, pp.225-235,2006.

18. H.Satoh, N.Niki, K.Eguchi, M.Kaneko, N. Moriyama, H.Ohmatsu, R.Kakinuma, H.Masuda, S.Machida, " Computer-aided diagnosis workstation and network system for chest diagnosis based on multislice CT images," SPIE Medical Imaging, San Diego,Vol.6516, pp.65161E-1-11,2007.

19. H.Satoh, N.Niki, K.Eguchi, M.Kaneko, N.Moriyama, H.Ohmatsu, R.Kakinuma, H.Masuda, S.Machida, " Computer-aided diagnosis workstation and network system for chest diagnosis based on multislice CT images," SPIE Medical Imaging, San Diego,Vol.6919, pp.691916-1-11,2008.

20. H.Satoh, N.Niki, K.Eguchi, M.Kaneko, N.Moriyama, H.Ohmatsu, R.Kakinuma, H.Masuda, S.Machida, "Computer-aided diagnosis workstation and network system for chest diagnosis based on multislice CT images," SPIE Medical Imaging, Orland, Vol.7264, pp.726410-1-12, 2009.

21. H.Satoh, N.Niki, K.Eguchi, M.Kaneko, N.Moriyama, H.Ohmatsu, R.Kakinuma, "Computer-aided diagnosis workstation and teleradiology network system for chest diagnosis using the web medical image conference system with a new information security solution,” SPIE Medical Imaging, San Diego, VOL.7628, pp.762813-1-12, 2010

22. Hitoshi Satoh, Noboru Niki, Kenji Eguchi,Hironobu Ohmatsu, Masahiro Kaneko, Ryutaro Kakinuma, Noriyuki Moriyama: Teleradiology network system and computer-aided diagnosis workstation using the web medical image conference system with a new information security solution, SPIE Medical Imaging, VOL.7967, pp.796711-1-12, 2011 